\title{
Appreciating the broad clinical features of SMAD4 mutation carriers: a multicenter chart review
}

\author{
Karen E. Wain, CGC ${ }^{1}$, Marissa S. Ellingson, CGC ${ }^{2}$, Jamie McDonald, LGC ${ }^{3}$, Amanda Gammon, CGC , \\ Maegan Roberts, $\mathrm{CGC}^{5}$, Pavel Pichurin, $\mathrm{MD}^{6}$, Ingrid Winship, $\mathrm{MBChB}, \mathrm{MD}^{7,8}$, \\ Douglas L. Riegert-Johnson, MD ${ }^{9}$, Jeffrey N. Weitzel, MD ${ }^{10}$ and Noralane M. Lindor, MD ${ }^{11}$
}

\begin{abstract}
Heterozygous loss-of-function SMAD4 mutations are associated with juvenile polyposis syndrome and hereditary hemorrhagic telangiectasia. Some carriers exhibit symptoms of both conditions, leading to juvenile polyposis-hereditary hemorrhagic telangiectasia syndrome. Three families have been reported with connective tissue abnormalities. To better understand the spectrum and extent of clinical findings in SMAD4 carriers, medical records of 34 patients (20 families) from five clinical practices were reviewed. Twenty-one percent of the patients $(7 / 34)$ had features suggesting a connective tissue defect: enlarged aortic root $(n=3)$, aortic and mitral insufficiency $(n=2)$, aortic dissection $(n=1)$, retinal detachment $(n=$ $1)$, brain aneurysms $(n=1)$, and lax skin and joints $(n=1)$. Juvenile polyposis-specific findings were almost uniformly present but variable. Ninety-seven percent of the patients had colon polyps that were generally pan-colonic and of variable histology and number. Forty-
\end{abstract}

Juvenile polyposis (JP; OMIM 174900) and hereditary hemorrhagic telangiectasia (HHT; OMIM 187300 and 600376) are distinct syndromes caused by mutations in genes within the transforming growth factor (TGF)- $\beta$ signaling pathway. Several published case reports, beginning in 1980, describe patients with features of both JP and HHT. ${ }^{1-6}$ Given the rarity of both syndromes and the existence of several reports of patients with features of both disorders, it was suspected that a distinct syndrome combining these two entities might exist. A combined syndrome of JP-HHT, caused by SMAD4 mutations, is now recognized as a distinct entity (OMIM 175050)..$^{7-13}$

JP is a genetically heterogeneous autosomal-dominant hamartomatous polyposis syndrome characterized by multiple gastrointestinal (GI) polyps of juvenile histology. Patients typically present in the first or second decade of life ${ }^{14}$ with symptoms including rectal bleeding, anemia, prolapse, abdominal pain, diarrhea, and intussusception. ${ }^{15,16}$ In general, polyps are typically distributed evenly throughout the colon and are less commonly found in the small intestine and stomach. ${ }^{14}$ However, there is a genotype-phenotype correlation between SMAD4 mutations and severe gastric polyposis. ${ }^{17-19}$ The total number of polyps varies greatly, from a few to hundreds. ${ }^{14}$ Historically, eight percent of the patients (15/31) had extensive gastric polyposis Hereditary hemorrhagic telangiectasia features, including epistaxis (19/31, 61\%), mucocutaneous telangiectases (15/31, 48\%), liver arteriovenous malformation $(6 / 16,38 \%)$, brain arteriovenous malformation $(1 / 26,4 \%)$, pulmonary arteriovenous malformation $(9 / 17$, $53 \%)$, and intrapulmonary shunting $(14 / 23,61 \%)$, were documented in $76 \%$ of the patients. SMAD4 carriers should be managed for juvenile polyposis and hereditary hemorrhagic telangiectasia because symptoms of both conditions are likely yet unpredictable. Connective tissue abnormalities are an emerging component of juvenile polyposis-hereditary hemorrhagic telangiectasia syndrome, and larger studies are needed to understand these manifestations.

Genet Med advance online publication 13 February 2014

Key Words: connective tissue; HHT; JP; SMAD4

the estimated lifetime risk for GI malignancies for individuals with JP has ranged from 39 to $68 \%$; however, this risk has been shown to be significantly reduced through increased surveillance. ${ }^{19-21}$ Approximately half of patients meeting clinical criteria for JP have identifiable mutations in the SMAD4 or $B M P R 1 A$ genes. ${ }^{22}$

HHT is also a genetically heterogeneous autosomal-dominant disorder. It leads to vascular dysplasia characterized by mucocutaneous telangiectases of the fingertips, lips, oral mucosa, and nose, and arteriovenous malformations (AVMs) of the lungs, brain, liver, GI system, and spine. ${ }^{23}$ Symptoms are not typically present at birth but develop with increasing age. ${ }^{23}$ The most common presenting symptom is epistaxis, occurring eventually in more than $90 \%$ of patients and beginning on average at the age of 12 years. ${ }^{24}$ Serious complications may result if AVMs are unrecognized and untreated, including anemia, hemorrhage, brain abscess, transient ischemic attack, seizure, and stroke. ${ }^{23}$ Approximately $80-85 \%$ of patients with a clinical diagnosis of HHT have a mutation identifiable in the $E N G$, ACVRL1 (ALK1), or SMAD4 genes. ${ }^{25}$

Recently, a small number of patients with SMAD4 mutations and the combined JP-HHT syndrome have been reported

\footnotetext{
${ }^{1}$ Laboratory Medicine and Pathology, Mayo Clinic, Rochester, Minnesota, USA; ${ }^{2}$ Center for Individualized Medicine, Mayo Clinic, Rochester, Minnesota, USA; ${ }^{3}$ Department of Pathology, University of Utah, Salt Lake City, Utah, USA; ${ }^{4}$ High Risk Cancer Clinics, Huntsman Cancer Institute, Salt Lake City, Utah, USA; ${ }^{5}$ GeneDx, Gaithersburg, Maryland, USA; ${ }^{6}$ Medical Genetics, Mayo Clinic, Rochester, Minnesota, USA; ${ }^{7}$ Department of Medicine, University of Melbourne, Melbourne, Australia; ${ }^{8}$ Genetic Medicine, Royal Melbourne Hospital, Melbourne, Australia; ${ }^{9}$ Medical Genetics and Gastroenterology, Mayo Clinic, Jacksonville, Florida, USA; ${ }^{10} \mathrm{Clinical}$ Cancer Genetics, City of Hope, Duarte, California, USA; ${ }^{11}$ Health Science Research, Mayo Clinic Scottsdale, Arizona, USA. Correspondence: Noralane M. Lindor (nlindor@mayo.edu)
} 
with various findings suggestive of connective tissue disorders, including aortic root dilation, thoracic aortic aneurysm, aortic dissection, skeletal features of Marfan syndrome, and mitral valve abnormalities. ${ }^{26,27}$ In addition, two individuals with SMAD4 mutations from a series of JP patients had arterial aneurysms, and three individuals (genotype not provided) had mitral valve prolapse. ${ }^{19}$ These findings have not been classically associated with the JP or HHT entities. All known genes causative of JP and HHT encode members of the TGF- $\beta$ signaling pathway. Mutations in several other genes within this pathway, such as TGFBR1, TGFBR2, TGFB2, and SMAD3, cause familial thoracic aortic aneurysms and aortic dissections, which provides a possible explanation for this reported connection. These recent reports provided the foundation for the hypothesis that individuals with SMAD4 mutations might be susceptible to a broader range of medical issues than their presenting clinical features might indicate.

Clinical management recommendations have been published for JP and $\mathrm{HHT},{ }^{28,29}$ but given the genetic heterogeneity involved, and the paucity of data on which recommendations are made, we sought to increase the understanding of the SMAD4 mutation phenotype by identifying a meaningful number of SMAD4 mutation carriers and reviewing their medical histories.

\section{MATERIALS AND METHODS}

A retrospective multicenter chart review of SMAD4 mutation carriers was conducted at five clinical practices. Patients were evaluated in a variety of clinical settings, including HHT centers and medical genetics, gastroenterology, and oncology departments. These sites included Mayo Clinic Rochester (Rochester, MN), Mayo Clinic Florida (Jacksonville, FL), Huntsman Cancer Institute and HHT Center, University of Utah (Salt Lake City, UT), City of Hope (Duarte, CA), and the Royal Melbourne Hospital (Melbourne, Australia). This study was approved by the Mayo Clinic institutional review board and individually at the other sites if required. Electronic medical records were searched from 1996 to 2011 using a variety of terms including SMAD4, juvenile polyposis, and hereditary hemorrhagic telangiectasia. The diagnostic database from the Department of Medical Genetics at Mayo Clinic Rochester was also reviewed to identify potential cases. Cases from Huntsman Cancer Institute were identified through the Hereditary Gastrointestinal Cancers Registry, and these patients had also undergone a clinical genetics evaluation at Huntsman Cancer Institute. The City of Hope Clinical Cancer Genetics Community Research Network includes a cross-section of cancer center and community-based clinics, and all genetic cancer risk assessment patients are invited to participate in an institutional review board-approved prospective registry at the time of consultation..$^{30}$

Inclusion criteria consisted of documentation of a pathogenic SMAD4 mutation and availability of the patient's medical records for review. Patients with Myhre syndrome (OMIM 139210; a disorder characterized by intellectual impairment, dysmorphic facial features, and skeletal anomalies and that is caused by gain-of-function mutations in one specific codon in the SMAD4 gene) were excluded.

A total of 34 mutation carriers from 20 families were included. A standardized detailed chart abstraction form was created, and data were abstracted from the medical records at each institution. Data collected included age at evaluation/procedure, the specific SMAD4 mutation identified, clinical features of JP and HHT, and results of specific screening tests. In addition to a careful focus on SMAD4-associated pathology, such as neoplasms and connective tissue findings, the abstraction process also provided the opportunity for investigators to review and collect information on other types of medical diagnoses. Due to the multicenter and retrospective nature of this study and to patient and provider variability, there was not a unified surveillance protocol followed for all patients.

\section{RESULTS}

Thirty-four SMAD4 mutation carriers from 20 families were identified and had charts available for review. The mean age at last visit was 35.1 years (range: $0.1-74$ years). Fourteen different mutations were identified; three mutations were reported in more than one family. Seven of these mutations have been reported previously in association with JP, JP-HHT, or HHT (see Supplementary Table S1 online). One individual is reportedly a member of a family originally investigated in the study published by Howe et al. ${ }^{31} \mathrm{He}$ had declined genetic testing but has a clinical diagnosis and is a presumed carrier for the c.13721375delACAG mutation.

Clinical findings are summarized in Table 1 . Twenty-one percent of the patients (7/34), from five separate families, had features of a connective tissue disorder, including enlarged aortic root $(n=3)$, aortic and mitral insufficiency $(n=2)$, fatal aortic dissection $(n=1)$, retinal detachment $(n=1)$, brain aneurysms $(n=1)$, and lax skin and joints $(n=1)$. These individuals were all between the ages of 42 and 65 years at the time of this study, although exact ages of onset for connective tissue findings were not noted. All five families had different mutations in various domains of the gene.

Lower GI polyps were reported in $97 \%$ of individuals who had undergone colonoscopy (31/32, identified at 4-51 years). Individuals underwent their first colon exam on average at the age of 23 years (range: 4-51 years) for one or more indications including anemia $(n=8)$, GI bleeding \pm anemia $(n=$ 12), known or suspected JP $(n=10)$, obstructive symptoms ( $n$ $=3$ ), and unknown condition $(n=5)$. The most recent colon exams were conducted at a mean age of 36 years (range: 6-73 years) and were completed within the preceding 3 years for all but three individuals. Polyps were generally pan-colonic and of variable histology. The total number of polyps ranged from 1 to more than 1,000 and did not appear to be associated with age; 1 individual (now 42 years of age) was only 17 years of age when more than 1,000 polyps were discovered. Polyps were present in all but one patient younger than 21 years who had a colonoscopy. When the number of polyps was available, $80 \%$ (16/20) had less than 100 polyps. Thirty-one individuals had 
Table 1 Clinical findings in SMAD4 mutation carriers

\begin{tabular}{|c|c|}
\hline Clinical findings & $\begin{array}{c}\text { Percentage and } \\
\text { number of individuals }\end{array}$ \\
\hline Colon polyps & $97 \%(31 / 32)$ \\
\hline Gastric polyps & $68 \%(21 / 31)$ \\
\hline Extensive polyposis & $48 \%(15 / 31)$ \\
\hline $1-10$ polyps & $19 \%(6 / 31)$ \\
\hline Small-bowel polyps & $39 \%(11 / 28)$ \\
\hline Neoplasms & $26 \%(9 / 34)$ \\
\hline Colorectal & $9 \%(3 / 34)$ \\
\hline Hurthle-cell thyroid & $6 \%(2 / 34)$ \\
\hline Ovarian teratomas & $3 \%(1 / 34)$ \\
\hline Lymphoma/jejunal & $3 \%(1 / 34)$ \\
\hline Esophageal adenocarcinoma & $3 \%(1 / 34)$ \\
\hline Pancreatic neoplasm & $3 \%(1 / 34$ \\
\hline Epistaxis & $61 \%(19 / 31)$ \\
\hline Telangiectases & $48 \%(15 / 31)$ \\
\hline \multicolumn{2}{|l|}{ AVMs } \\
\hline Brain & $4 \%(1 / 26)$ \\
\hline Lung & $53 \%(9 / 17)$ \\
\hline Liver & $38 \%(6 / 16)$ \\
\hline Intrapulmonary shunt & $61 \%(14 / 23)$ \\
\hline \multicolumn{2}{|c|}{ HHT-related symptoms in absence of: } \\
\hline AVM/IP shunt & $15 \%(5 / 34)$ \\
\hline Migraines & $12 \%(4 / 34)$ \\
\hline Seizure & $3 \%(1 / 34)$ \\
\hline Connective tissue finding & $21 \%(7 / 34)$ \\
\hline Enlarged aortic root & $9 \%(3 / 34)$ \\
\hline Aortic/mitral insufficiency & $6 \%(2 / 34)$ \\
\hline Aortic dissection & $3 \%(1 / 34)$ \\
\hline Retinal detachment & $3 \%(1 / 34)$ \\
\hline Brain aneurysms & $3 \%(1 / 34)$ \\
\hline Lax skin and joints & $3 \%(1 / 34)$ \\
\hline
\end{tabular}

AVM, arteriovenous malformation; $\mathrm{HHT}$, hereditary hemorrhagic telangiectasia; $\mathrm{IP}$, intrapulmonary.

gastric imaging and 28 had small-bowel imaging, typically by upper endoscopy, with first exams performed at a mean age of 31 years (range: 4-73 years) and 37 years (range: 4-72 years), respectively. The most recent gastric and small-bowel imaging studies had been performed within the preceding year for all but one individual. Indications for gastric and small-bowel screening included bleeding, pain, or another physical symptom $(n=10)$; screening based on the presence of colon polyps or genetic test results $(n=16)$; or a family history of JP $(n=6)$. Forty-eight percent of the patients (15/31, identified at 26-59 years of age) had extensive gastric polyposis (often described as "carpeted" or "innumerable"), 19\% (6/31, identified at 17-63 years of age) had 1-10 gastric polyps of variable histology, and $32 \%(10 / 31)$ had no gastric polyps. Small-bowel polyps were identified in $39 \%$ of patients $(11 / 28$, range: $1-10$, identified at 28-52 years of age) and were duodenal in eight individuals and duodenal and jejunal in one individual; the sites were not specified for two individuals. One individual had diffuse jejunal thickening at the age of 58 years.
Malignancies were reported in eight individuals and included three individuals with colorectal cancers (diagnosed at the ages of 22, 30, and 38 years), two with Hurthle-cell thyroid tumors (ages: 21 and 42 years), one with bilateral metachronous malignant ovarian teratomas (ages: 19 and 38 years), one with lymphoma (age: 55 years) and jejunal cancer (age: 65 years), and one with adenocarcinoma of the esophagus arising in a region of Barrett esophagus (age: 39 years). One mucinous neoplasm with low-grade dysplasia in the pancreatic head was reported (age not provided). The only malignancies that led to diagnosis and subsequent screening were the three colorectal cancers. The jejunal cancer was found due to new symptoms in an individual who was already receiving colon screening. The remaining malignancies appear to have been identified incidentally. In addition, 15 individuals had documented surgical procedures, including 10 with some type of colectomy or colon resection (2 for cancer, 7 for polyposis, and 1 for polyposis and Crohn disease) and 7 with gastrectomy due to either extensive polyposis or the presence of very large polyps. Gastric screening was frequently initiated due to new symptoms or family history information in individuals already receiving early colon screening.

Features associated with HHT were documented in $76 \%$ of individuals and included epistaxis $(19 / 31 ; 61 \%$, all deemed abnormal in childhood), mucocutaneous telangiectases (15/31; 48\%, identified at 5-65 years of age), liver AVM (6/16; $38 \%$, identified at $21-52$ years of age), brain AVM $(1 / 26 ; 4 \%$, identified at 52 years of age), pulmonary AVM (9/17; 53\%, identified at birth to 52 years of age), and intrapulmonary shunting on bubble echocardiogram $(14 / 23$; $61 \%$, identified at 5-59 years of age). Eight of the nine individuals under the age of 21 years had some screening for HHT features (two of these had only brain magnetic resonance imaging, whereas the remaining had brain magnetic resonance imaging and echocardiogram/chest computed tomography), and 63\% (5/8) had HHT features. GI bleeding was documented for $83 \%$ of the individuals with iron deficiency (20/24). All of these individuals had known GI polyps, so it is unclear whether anemia was due to polyps or bleeding from telangiectases. In addition, 50\% of patients (17/34), five of whom had no detectable visceral AVMs by computed tomography or intrapulmonary shunting, were noted to have migraines $(n=9)$, exercise intolerance $(n=$ 6 ), and/or digital clubbing $(n=3)$, symptoms frequently found in patients with HHT.

\section{DISCUSSION}

The clinical manifestations of JP were present in this cohort of SMAD4 mutation carriers, the largest to date, with frequencies and characteristics that are quite consistent with the published literature. ${ }^{1}$ Colonic polyps were present in $97 \%$ of individuals who had undergone colonoscopy; the one individual with no colonic polyps was 7 years old at the time of last colonoscopy. As expected, gastric and small-bowel polyps were less frequent than colonic polyps. However, we observed extensive gastric polyposis in $48 \%$ of individuals who had this screening, which is consistent with previous studies showing that extensive 
gastric polyposis is more common with SMAD4 mutations as compared with BMPR1A mutations. ${ }^{17-19}$ HHT symptoms were also observed at rates that are near the expected frequencies for individuals with HHT, although some individuals may not have met Curacao criteria for clinical diagnosis. ${ }^{67}$ Epistaxis and mucocutaneous telangiectases, in particular, were documented relatively less frequently in this cohort than is typical for HHT patients, 61 and $48 \%$, respectively. This could certainly be due in part to age-related penetrance, as nine of the individuals who were assessed for these symptoms were younger than 21 years of age or because the patients were under the care of physicians who were focused on JP rather than HHT.

Previous studies of SMAD4 mutation carriers are consistent with the results of this study in that features of JP and HHT are regularly present. In 2004, Gallione et al. ${ }^{7}$ tested 14 patients from 7 families with features of both JP and HHT for mutations in the ENG, ACVRL1, and SMAD4 genes. Mutations were identified in SMAD4 in all 14 patients. The SMAD4 mutations cosegregated with the JP-HHT phenotype. A subsequent case series, in 2010, describing the clinical features of JP-HHT included 19 patients, 15 of whom had identifiable SMAD4 mutations. ${ }^{9}$ All 15 SMAD4 mutation carriers exhibited features of both JP and HHT, the most frequent being juvenile polyps (15/15), pulmonary AVMs (13/15), epistaxis (13/15), telangiectases (10/15), and anemia (9/15). The most recent series, by O'Malley et al., ${ }^{13}$ reports SMAD4 mutations in 21 individuals from nine families who had a diagnosis of JP. Seventeen of these individuals underwent HHT screening; 94\% $(n=16)$ of these met clinical criteria for HHT, and $6 \%(n=1)$ had a suspected diagnosis.

The rate of malignant neoplasms in our cohort was $24 \%$, which is lower than previous reports and may be due to the young ages of some individuals as well as more frequent screening or prophylactic surgery, such as colectomy and gastrectomy. Five individuals had neoplasms previously reported in association with SMAD4 mutations (three colorectal, one jejunal, and one pancreatic). The one patient with esophageal adenocarcinoma also had Barrett esophagus, the major risk factor for this type of cancer. ${ }^{32}$ To our knowledge, germ line SMAD4 mutations have not previously been reported in association with Hurthle-cell thyroid tumors, ovarian teratomas, or lymphomas. However, acquired changes in SMAD4 expression and the TGF- $\beta$ pathway have been implicated in tumorigenesis and/or progression in papillary thyroid carcinoma, ovarian carcinoma, and some lymphomas. ${ }^{33-38}$ Somatic mutations and loss of heterozygosity have also been frequently found in breast cancers, and one recent study reported intronic germ line SMAD4 variants in BRCA1/2-negative breast cancer patients. ${ }^{39}$ Larger studies are needed to determine if additional cancer risks may be present for SMAD4 mutation carriers.

Connective tissue findings have recently been reported in three families with SMAD4 mutations as well as in a few individuals from a JP cohort. ${ }^{19,26,27}$ These include mitral valve prolapse, aortic root dilatation, thoracic aortic dissections, and some skeletal features suggestive of Marfan syndrome. There have also been rare reports of individuals with HHT and large aortic aneurysms, ${ }^{40,41}$ and a genetic mutation (in ACVRL1) was reported for only one of them. ${ }^{40}$ Seven of the 34 individuals in the current study (21\%), from five separate families, had documented evidence of connective tissue findings with some features overlapping those of previous reports. In addition, our results include reports of a retinal detachment, brain aneurysms (in the absence of cerebral AVMs), and lax skin and joints, which have not been reported previously.

All of the genes associated with HHT (ENG, ACVRL1, and $S M A D 4)$ are part of the TGF- $\beta$ signaling pathway, which is essential for vascular integrity and angiogenic remodeling. ${ }^{42}$ In addition, SMAD4 has the distinction of also acting as a tumor suppressor, which explains the JP phenotype. ${ }^{43}$ Although the newly reported connective tissue findings have not classically been considered to be manifestations of JP-HHT, perturbations of the TGF- $\beta$ pathway due to mutations in other genes, such as FBN1, TGFBR1, TGFBR2, TGFBR, SMAD3, MYH11, and ACTA2, are well-described causes of several connective tissue disorders, such as Marfan syndrome, familial thoracic aortic aneurysms and aortic dissections, and Loeys-Dietz syndrome. ${ }^{44,45}$ Therefore, there is biological plausibility for observing these additional connective tissue findings, and further studies are needed to more formally assess these risks in SMAD4 mutation carriers.

The findings of this study strengthen the argument that all SMAD4 mutation carriers should be screened following both JP and HHT protocols and that echocardiography, which is already used with injected saline to screen for pulmonary AVMs, is doubly beneficial to assess for enlarged aortic roots. ${ }^{9,13}$ Patients with Loeys-Dietz syndrome and other TGF- $\beta$-associated connective tissue disorders are now treated with angiotensin receptor blockers for primary prevention of vascular complications. Although mechanistically attractive, no empiric data support the use of angiotensin receptor blockers in SMAD4 mutation carriers. More rigorously conducted studies are needed to quantify the risks, the possible benefits of screening, and the efficacy of angiotensin receptor blockers and other therapies. Finally, these results underscore the importance of appreciating the impact of a patient's age in assessing phenotype. Age-related penetrance is a well-documented phenomenon associated with HHT features. Although colonic polyps can frequently be found in the first decade of life, there is a wide range of onset, and additional studies are needed to better determine the range of onset for gastric and small-bowel polyps.

The individuals in this study were ascertained based on SMAD4 carrier status, as opposed to a clinical diagnosis of JP and/or HHT, and the majority of them had screening for manifestations of both conditions. However, this study is still limited by a relatively small sample size, the young ages of some individuals, and incomplete screening for all findings of interest in some individuals. In particular, connective tissue findings may not have been recognized or documented adequately in the medical records due to a lack of clinical suspicion, so we consider the proportion manifesting those 
findings as the lowest possible estimate for prevalence in JP-HHT.

\section{Conclusion}

This multicenter retrospective chart review of 34 individuals with SMAD4 mutations associated with JP-HHT provides additional documentation of the clinical phenotypic spectrum associated with these mutations. Regardless of initial clinical presentation, the majority of individuals had clinical features of both JP and HHT. Findings typically associated with TGF- $\beta$ connective tissue disorders were observed in $21 \%$ of individuals with SMAD4 mutations. Complete JP and HHT clinical screening is recommended for all patients with SMAD4 mutations, and clinicians should have a high suspicion for aortic root dilatation, thoracic aortic aneurysms, or other connective tissue pathology in these patients.

\section{SUPPLEMENTARY MATERIAL}

Supplementary material is linked to the online version of the paper at http://www.nature.com/gim

\section{ACKNOWLEDGMENTS}

The authors thank Brittany Thomas for her assistance with the manuscript. The authors acknowledge the use of core facilities supported by grant P30 CA042014, awarded to Huntsman Cancer Institute. Case finding at City of Hope was supported by award 1RC4CA153828 from the US National Cancer Institute (principal investigator: J.N.W.).

\section{DISCLOSURE}

The authors declare no conflict of interest.

\section{REFERENCES}

1. Cox KL, Frates RC Jr, Wong A, Gandhi G. Hereditary generalized juvenile polyposis associated with pulmonary arteriovenous malformation. Gastroenterology 1980;78:1566-1570.

2. Baert AL, Casteels-Van Daele M, Broeckx J, Wijndaele L, Wilms G, Eggermont $\mathrm{E}$. Generalized juvenile polyposis with pulmonary arteriovenous malformations and hypertrophic osteoarthropathy. AJR Am J Roentgenol 1983:141:661-662.

3. Prieto G, Polanco I, Sarria J, Larrauri J, Lassaletta L. Association of juvenile and adenomatous polyposis with pulmonary arteriovenous malformation and hypertrophic osteoarthropathy. J Pediatr Gastroenterol Nutr 1990;11:133-137.

4. Schumacher B, Frieling T, Borchard F, Hengels KJ. Hereditary hemorrhagic telangiectasia associated with multiple pulmonary arteriovenous malformations and juvenile polyposis. Z Gastroenterol 1994;32:105-108.

5. Radin DR. Hereditary generalized juvenile polyposis: association with arteriovenous malformations and risk of malignancy. Abdom Imaging 1994;19:140-142.

6. Inoue $\mathrm{S}$, Matsumoto $\mathrm{T}$, lida $\mathrm{M}$, et al. Juvenile polyposis occurring in hereditary hemorrhagic telangiectasia. Am J Med Sci 1999;317:59-62.

7. Gallione CJ, Repetto GM, Legius E, et al. A combined syndrome of juvenile polyposis and hereditary haemorrhagic telangiectasia associated with mutations in MADH4 (SMAD4). Lancet 2004;363:852-859.

8. Gallione CJ, Richards JA, Letteboer TG, et al. SMAD4 mutations found in unselected HHT patients. J Med Genet 2006;43:793-797.

9. Gallione $C$, Aylsworth AS, Beis J, et al. Overlapping spectra of SMAD4 mutations in juvenile polyposis (JP) and JP-HHT syndrome. Am J Med Genet $A$ 2010;152A:333-339.

10. Iyer NK, Burke CA, Leach BH, Parambil JG. SMAD4 mutation and the combined syndrome of juvenile polyposis syndrome and hereditary haemorrhagic telangiectasia. Thorax 2010;65:745-746.
11. Schwenter F, Faughnan ME, Gradinger AB, et al. Juvenile polyposis, hereditary hemorrhagic telangiectasia, and early onset colorectal cancer in patients with SMAD4 mutation. J Gastroentero/ 2012;47:795-804.

12. Sumitomo $Y$, Yamashita $Y$, Itai $R$, et al. [A case of combined syndrome of juvenile polyposis and hereditary hemorrhagic telangiectasia associated with SMAD4 mutation]. Nihon Shokakibyo Gakkai Zasshi 2013;110:64-73.

13. O'Malley M, LaGuardia L, Kalady MF, et al. The prevalence of hereditary hemorrhagic telangiectasia in juvenile polyposis syndrome. Dis Colon Rectum 2012;55:886-892.

14. Desai DC, Neale KF, Talbot IC, Hodgson SV, Phillips RK. Juvenile polyposis. Br J Surg 1995;82:14-17.

15. Veale AM, McColl I, Bussey HJ, Morson BC. Juvenile polyposis coli. J Med Genet 1966;3:5-16

16. Coburn MC, Pricolo VE, DeLuca FG, Bland KI. Malignant potential in intestinal juvenile polyposis syndromes. Ann Surg Oncol 1995;2:386-391.

17. Aretz $S$, Stienen $D$, Uhlhaas $S$, et al. High proportion of large genomic deletions and a genotype phenotype update in 80 unrelated families with juvenile polyposis syndrome. J Med Genet 2007;44:702-709.

18. Friedl W, Uhlhaas S, Schulmann K, et al. Juvenile polyposis: massive gastric polyposis is more common in MADH4 mutation carriers than in BMPR1A mutation carriers. Hum Genet 2002;111:108-111.

19. Latchford AR, Neale K, Phillips RK, Clark SK. Juvenile polyposis syndrome: a study of genotype, phenotype, and long-term outcome. Dis Colon Rectum 2012;55:1038-1043.

20. Howe JR, Mitros FA, Summers RW. The risk of gastrointestinal carcinoma in familial juvenile polyposis. Ann Surg Oncol 1998;5:751-756.

21. Brosens LA, Langeveld D, van Hattem WA, Giardiello FM, Offerhaus GJ. Juvenile polyposis syndrome. World J Gastroenterol 2011;17:4839-4844.

22. Calva-Cerqueira D, Chinnathambi S, Pechman B, Bair J, Larsen-Haidle J, Howe JR. The rate of germline mutations and large deletions of SMAD4 and BMPR1A in juvenile polyposis. Clin Genet 2009;75:79-85.

23. Begbie ME, Wallace GM, Shovlin CL. Hereditary haemorrhagic telangiectasia (Osler-Weber-Rendu syndrome): a view from the $21^{\text {st }}$ century. Postgrad Med J 2003;79:18-24.

24. AAssar OS, Friedman CM, White RI Jr. The natural history of epistaxis in hereditary hemorrhagic telangiectasia. Laryngoscope 1991;101:977-980.

25. Prigoda NL, Savas S, Abdalla SA, et al. Hereditary haemorrhagic telangiectasia: mutation detection, test sensitivity and novel mutations. J Med Genet 2006;43:722-728.

26. Andrabi S, Bekheirnia MR, Robbins-Furman P, Lewis RA, Prior TW, Potocki L. SMAD4 mutation segregating in a family with juvenile polyposis, aortopathy, and mitral valve dysfunction. Am J Med Genet A 2011;155A:1165-1169.

27. Teekakirikul P, Milewicz DM, Miller DT, et al. Thoracic aortic disease in two patients with juvenile polyposis syndrome and SMAD4 mutations. Am J Med Genet A 2013;161A:185-191.

28. Faughnan ME, Palda VA, Garcia-Tsao G, et al.; HHT Foundation International - Guidelines Working Group. International guidelines for the diagnosis and management of hereditary haemorrhagic telangiectasia. J Med Genet 2011:48:73-87.

29. Larsen Haidle J, Howe JR. Juvenile Polyposis Syndrome. In: Pagon RA, Adam MP, Bird TD, Dolan CR, Fong CT, Stephens K (eds). GeneReviews. Seattle, WA, 1993. http://www.ncbi.nlm.nih.gov/books/NBK1469.

30. MacDonald DJ, Blazer KR, Weitzel JN. Extending comprehensive cancer center expertise in clinical cancer genetics and genomics to diverse communities: the power of partnership. J Natl Compr Canc Netw 2010;8: 615-624.

31. Howe JR, Roth S, Ringold JC, et al. Mutations in the SMAD4/DPC4 gene in juvenile polyposis. Science 1998;280:1086-1088.

32. Spechler SJ. Barrett esophagus and risk of esophageal cancer: a clinical review. JAMA 2013;310:627-636.

33. D'Inzeo S, Nicolussi A, Ricci A, et al. Role of reduced expression of SMAD4 in papillary thyroid carcinoma. J Mol Endocrino/ 2010;45:229-244.

34. D'Inzeo S, Nicolussi A, Donini CF, et al. A novel human Smad4 mutation is involved in papillary thyroid carcinoma progression. Endocr Relat Cancer 2012;19:39-55

35. Eloy C, Santos J, Cameselle-Teijeiro J, Soares P, Sobrinho-Simões M. TGFbeta/Smad pathway and BRAF mutation play different roles in circumscribed and infiltrative papillary thyroid carcinoma. Virchows Arch 2012;460:587600.

36. Chen C, Sun MZ, Liu S, et al. Smad4 mediates malignant behaviors of human ovarian carcinoma cell through the effect on expressions of E-cadherin, plasminogen activator inhibitor-1 and VEGF. BMB Rep 2010;43:554-560. 
37. Yeh KT, Chen TH, Yang HW, et al. Aberrant TGFB/SMAD4 signaling contributes to epigenetic silencing of a putative tumor suppressor, RunX1T1 in ovarian cancer. Epigenetics 2011;6:727-739.

38. Go JH. Altered expression of Smad proteins in T or NK-cell lymphomas. Cancer Res Treat 2008;40:197-201.

39. Tram E, Ibrahim-Zada I, Briollais L, Knight JA, Andrulis IL, Ozcelik H. Identification of germline alterations of the mad homology 2 domain of SMAD3 and SMAD4 from the Ontario site of the breast cancer family registry (CFR). Breast Cancer Res 2011;13:R77.

40. Andersen ND, Dubose J, Shah A, Lee T, Wechsler SB, Hughes GC. Thoracic endografting in a patient with hereditary hemorrhagic telangiectasia presenting with a descending thoracic aneurysm. J Vasc Surg 2010;51: 468-470.

41. Hsi DH, Ryan GF, Hellems SO, Cheeran DC, Sheils LA. Large aneurysms of the ascending aorta and major coronary arteries in a patient with hereditary hemorrhagic telangiectasia. Mayo Clin Proc 2003;78:774-776.

42. Fernández-L A, Sanz-Rodriguez F, Blanco FJ, Bernabéu C, Botella LM. Hereditary hemorrhagic telangiectasia, a vascular dysplasia affecting the TGF-beta signaling pathway. Clin Med Res 2006;4:66-78.

43. Yang G, Yang X. Smad4-mediated TGF-beta signaling in tumorigenesis. Int J Bio/ Sci 2010;6:1-8.

44. Jondeau G, Boileau C. Genetics of thoracic aortic aneurysms. Curr Atheroscler Rep 2012;14:219-226.

45. Renard M, Callewaert B, Baetens M, et al. Novel MYH11 and ACTA2 mutations reveal a role for enhanced TGFB signaling in FTAAD. Int J Cardiol 2013;165:314-321.

46. Houlston R, Bevan S, Williams A, et al. Mutations in DPC4 (SMAD4) cause juvenile polyposis syndrome, but only account for a minority of cases. Hum Mol Genet 1998;7:1907-1912.
47. Woodford-Richens K, Bevan S, Churchman M, et al. Analysis of genetic and phenotypic heterogeneity in juvenile polyposis. Gut 2000;46:656-660.

48. Woodford-Richens KL, Rowan AJ, Poulsom R, et al. Comprehensive analysis of SMAD4 mutations and protein expression in juvenile polyposis: evidence for a distinct genetic pathway and polyp morphology in SMAD4 mutation carriers. Am J Pathol 2001;159:1293-1300.

49. Woodford-Richens KL, Rowan AJ, Gorman P, et al. SMAD4 mutations in colorectal cancer probably occur before chromosomal instability, but after divergence of the microsatellite instability pathway. Proc Natl Acad Sci USA 2001;98:9719-9723.

50. Kim IJ, Ku JL, Yoon KA, et al. Germline mutations of the dpc4 gene in Korean juvenile polyposis patients. Int J Cancer 2000;86:529-532.

51. van Hattem WA, Brosens LA, de Leng WW, et al. Large genomic deletions of SMAD4, BMPR1A and PTEN in juvenile polyposis. Gut 2008; 57:623-627.

52. Carr JC, Dahdaleh FS, Wang D, Howe JR. Germline mutations in SMAD4 disrupt bone morphogenetic protein signaling. J Surg Res 2012;174:211-214.

53. Howe JR, Sayed MG, Ahmed AF, et al. The prevalence of MADH4 and BMPR1A mutations in juvenile polyposis and absence of BMPR2, BMPR1B, and ACVR1 mutations. J Med Genet 2004;41:484-491.

54. Howe JR, Shellnut J, Wagner B, et al. Common deletion of SMAD4 in juvenile polyposis is a mutational hotspot. Am J Hum Genet 2002;70:1357-1362.

55. Pintiliciuc OG, Heresbach D, de-Lajarte-Thirouard AS, et al. Gastric involvement in juvenile polyposis associated with germline SMAD4 mutations: an entity characterized by a mixed hypertrophic and polypoid gastropathy. Gastroenterol Clin Biol 2008;32(5 Pt 1):445-450.

56. Sweet K, Willis J, Zhou XP, et al. Molecular classification of patients with unexplained hamartomatous and hyperplastic polyposis. JAMA 2005;294:2465-2473. 